\title{
A Real-world Prospective Study of Mother-to-child Transmission of HBV in China Using a Mobile Health Application (Shield 01)
}

\author{
Xueru Yin ${ }^{1}$, Guorong Han ${ }^{2}$, Hua Zhang ${ }^{3}$, Mei Wang ${ }^{4}$, Wenjun Zhang ${ }^{5}$, Yunfei Gao ${ }^{6}$, Mei Zhong ${ }^{6}$, \\ Xiaolan Wang ${ }^{7}$, Xiaozhu Zhong ${ }^{8}$, Guojun Shen ${ }^{9}$, Chuangguo Yang ${ }^{10}$, Huiyuan Liu ${ }^{11}$ \\ Zhihong Liu ${ }^{1}$, Po-Lin Chan ${ }^{12}$, Marc Bulterys ${ }^{13}$, Fuqiang Cui ${ }^{14}$, Hui Zhuang ${ }^{15}$, Zhihua Liu ${ }^{1 *}$ \\ and Jinlin $\mathrm{Hou}^{1 *}$
}

\begin{abstract}
${ }^{1}$ Department of Infectious Diseases, Nanfang Hospital, Southern Medical University, Guangzhou, Guangdong, China;
${ }^{2}$ Department of Gynecology and Obstetrics, The Second Affiliated Hospital of the Southeast University, Nanjing, Jiangsu, China; ${ }^{3}$ Department of Obstetrics and Gynecology, Beijing Youan Hospital, Capital Medical University, Beijing, China; ${ }^{4}$ Department of Obstetrics and Gynecology, The Fifth Medical Centre, Chinese People's Liberation Army General Hospital, Beijing, China; ${ }^{5}$ Department of Obstetrics and Gynecology, The Fifth Affiliated Hospital of Southern Medical University, Guangzhou, Guangdong, China; ${ }^{6}$ Department of Obstetrics and Gynecology, Nanfang Hospital, Southern Medical University, Guangzhou, Guangdong, China; ${ }^{7}$ Department of Obstetrics, Jiujiang Maternal and Child Care Center, Jiujiang, Jiangxi, China; ${ }^{8}$ Department of Infectious Diseases, Zhujiang Hospital, Southern Medical University, Guangzhou, Guangdong, China; ${ }^{9}$ Department of Infectious Diseases, Third People's Hospital of Jiujiang, Jiujiang, Jiangxi, China; ${ }^{10}$ Department of Infectious Diseases, The Third Affiliated Hospital of Southern Medical university, Guangzhou, Guangdong, China; ${ }^{11}$ Department of Severe Liver Diseases, Guangzhou Eighth People's Hospital, Guangzhou, Guangdong, China; ${ }^{12}$ World Health Organization/Western Pacific Regional Office (WPRO), Manila, Philippines; ${ }^{13}$ Global Hepatitis Programme, World Health Organization, Geneva, Switzerland; ${ }^{14}$ School of Public Health, Peking University, Beijing, China; ${ }^{15}$ Department of Microbiology and Center of Infectious Diseases, School of Basic Medical Sciences,
\end{abstract} Peking University Health Science Center, Beijing, China

\begin{abstract}
Background and Aims: The World Health Organization (WHO) Western Pacific Region set a target of eliminating mother-to-child transmission (MTCT) of hepatitis B virus (HBV) by 2030 . To assess the feasibility of this target in China, we carried out an epidemiological study to investigate the status quo of MTCT in the real-world setting. Methods: One thousand and eight hepatitis B surface antigen-positive pregnant women were enrolled at 10 hospitals. Immunoprophylaxis was administered to infants. In addition, mothers with HBV DNA level $>2,000,000 \mathrm{IU} / \mathrm{mL}$ were advised to initiate antiviral therapy during late pregnancy. A health application called SHIELD was used to manage the study. Results: Nine hundred and five of the enrolled mothers, with 924 infants, completed the follow-up. Birth-dose hepatitis $B$ vaccine and hepatitis B immunoglobulin were received by $99.7 \%$ and $99.7 \%$ of infants, respectively, within $24 \mathrm{~h}$ after birth. There
\end{abstract}

Keywords: Mother-to-child transmission; Hepatitis B virus; Antiviral therapy; Immunoprophylaxis; Shield Project.

Abbreviations: $\mathrm{HBeAg}$, hepatitis $\mathrm{B}$ e antigen; $\mathrm{HBIG}$, hepatitis $\mathrm{B}$ immunoglobulin $\mathrm{HBsAg}$, hepatitis B surface antigen; HBV, hepatitis B virus; HepB, hepatitis $B$ vaccine; LAM, lamivudine; LdT, telbivudine; MTCT, mother-to-child transmission; PVST, post-vaccination serologic testing; TDF, tenofovir disoproxil fumarate; WHO, World Health Organization.

Received: 4 December 2019; Revised: 21 January 2020; Accepted: 15 February 2020

*Correspondence to: Zhihua Liu, Hepatology Unit, Nanfang Hospital, Southern Medical University, Guangzhou, Guangdong 510515, China. Tel: +86-2062787432, Fax: +86-20-62786530, E-mail: zhihualiu@126.com; Jinlin Hou, Hepatology Unit, Nanfang Hospital, Southern Medical University, Guangzhou, Guangdong, 510515, China. Tel: +86-20-61641941, Fax: +86-20-62786530, E-mail: jlhousmu@163.com were 446 mothers who received antiviral therapy, including $72.3 \%$ of the mothers with HBV DNA level $>2,000,000 \mathrm{IU} / \mathrm{mL}$ and $21.0 \%$ of the mothers with HBV DNA level $<2,000,000$ $\mathrm{IU} / \mathrm{mL}$. Eight infants were infected with HBV. The overall rate of MTCT was $0.9 \%$. Birth defects were rare $(0.5 \%$ among infants with maternal antiviral exposure versus $0.7 \%$ among infants without exposure; $p=1.00)$. Conclusions:The MTCT rate was lower than the WHO Western Pacific Region elimination MTCT target in this real-world study, indicating that a comprehensive management composed of immunoprophylaxis to infants and antiviral prophylaxis to mothers may be a feasible strategy to achieve the 2030 WHO elimination goal. Citation of this article: Yin $X$, Han $G$, Zhang $H$, Wang $M$, Zhang W, Gao $Y$, et al. A real-world prospective study of mother-to-child transmission of HBV in China using a mobile health application (Shield 01). J Clin Transl Hepatol 2020;8(1):1-8. doi: 10.14218/JCTH.2019.00057.

\section{Introduction}

Mother-to-child transmission (MTCT) is the major mode of hepatitis B virus (HBV) exposure in areas with a high prevalence of HBV infection. ${ }^{1}$ MTCT among children born to hepatitis $B$ surface antigen ( $\mathrm{HBsAg}$ )-positive mothers will induce high rates of chronic HBV infection and is associated with high rates of severe clinical outcomes, such as liver cirrhosis, liver failure and hepatocellular carcinoma. 2,3

Combined immunoprophylaxis, including hepatitis $B$ vaccine (HepB) birth dose and hepatitis $B$ immunoglobulin (HBIG), administered within $24 \mathrm{~h}$ of birth to infants born to 
HBsAg-positive mothers is the most effective measure for preventing MTCT of HBV. ${ }^{4,5}$ However, despite immunoprophylaxis, MTCT cannot be completely prevented, and immunoprophylaxis fails in $2-5 \%$ of infants born to mothers with high levels of HBV DNA. ${ }^{6-8}$

In 2017, the Regional Framework for Eliminating MTCT of Human Immunodeficiency Virus, Hepatitis B and Syphilis in Asia and the Pacific, 2018-2030 (Triple elimination) proposed an integrated and coordinated approach towards elimination of these three infections. ${ }^{9}$ The framework adopts the target of $0.1 \% \mathrm{HBsAg}$ prevalence among children by 2030 , as set by the Global Health Sector Strategy on Viral Hepatitis 20162021. It also suggests antiviral therapy for mothers with high viral load for the Regional Framework for Eliminating MTCT of hepatitis $B$, building upon previous successful hepatitis $B$ vaccination programs.

In China, the HBV infection rate has been significantly decreasing since the universal immunization of newborns was initiated. ${ }^{10}$ The latest epidemiological survey (in 2014) showed that the HBsAg prevalence was as low as $0.32 \%$ among children under 5 years-old, as compared to $9.67 \%$ in 1992. ${ }^{11}$ China has a large population of HBsAg-positive pregnant women. It was reported that there were approximately one million infants born to $\mathrm{HBsAg}$-positive mothers in $2015 .{ }^{12}$ It is a great challenge, however, to decrease the HBsAg prevalence further in children under 5 years-old in order to achieve the World Health Organization (WHO) 2030 goal.

In the past decade, antiviral therapy during late pregnancy in mothers with high HBV DNA has been shown to be effective in preventing MTCT of $\mathrm{HBV}^{7,8,13-15}$ and is recommended by hepatitis $B$ guidelines for the management of pregnant women with chronic hepatitis B. ${ }^{1,16-18}$ In previous studies, no infant was infected with HBV in the group of hepatitis B e antigen $(\mathrm{HBeAg})$-positive mothers receiving antiviral therapy during late pregnancy, ${ }^{7,8}$ which indicates that elimination of HBV MTCT is possible with routine immunoprophylaxis for all infants and additional antiviral therapy for HBsAg-positive mothers with high viral load.

Since 2007, China established a rigorous strategy for prevention of HBV MTCT that requires immunization (timely HepB birth dose and HBIG plus two additional doses of HepB), and in 2010, universal screening of HBV in pregnant women was started. Given the increasing evidence of the effectiveness of maternal antiviral therapy in preventing MTCT, we initiated the Shield Project across China - aiming towards zero HBV transmission rate in infants born to $\mathrm{HBsAg}$-positive mothers. ${ }^{19}$

\section{Methods}

\section{Study design and population}

This was a prospective, multicenter, observational cohort study to investigate the status quo of MTCT of HBV in the real-world setting (ClinicalTrials.gov No. NCT03539016). The study was conducted between July 2015 and May 2018 in 10 hospitals in China. The subjects included pregnant women at gestational weeks 4-32 and their infants. The main inclusion criteria were pregnant women with positive HBsAg test for a minimum of six months, good compliance, and they and their infants could be followed-up as planned. Pregnant women were excluded if they had a positive serologic test for human immunodeficiency virus or hepatitis $C$ virus, any comorbidity that might reduce compliance, or if they were unable or unwilling to use the mobile health application. Immunoprophylaxis was administered to infants following the national Chinese vaccination schedule, i.e. HBIG and first dose of hepatitis B vaccine within $12 \mathrm{~h}$ after delivery and the other two doses of hepatitis $B$ vaccine at 1 and 6 months of age. In addition, mothers with HBV DNA level $>2,000,000 \mathrm{IU} / \mathrm{mL}$ were advised to initiate antiviral therapy during late pregnancy. Enrolled mothers and infants were prospectively followed until infant post-vaccination serologic testing (PVST) was performed at 7-12 months of age.

\section{Data collection and laboratory testing}

The information of the enrolled mothers, including their demographic data, antiviral treatment history, pregnancy and labor history, and comorbidities, were collected from prenatal medical records. HBV serologic markers, including $\mathrm{HBsAg}$, antibody to $\mathrm{HBsAg}, \mathrm{HBeAg}$, antibody to $\mathrm{HBeAg}$ and antibody to hepatitis B core antigen, HBV DNA levels, alanine aminotransferase, aspartate aminotransaminase, total bilirubin, direct bilirubin, and albumin were measured at baseline, at gestational week 28 , and at delivery for all enrolled mothers. Additional tests included liver ultrasound at baseline and PVST for infants at 7-12 months of age. The mode of delivery, neonatal characteristics (height, weight, head circumference, Apgar score, and birth defect) and breastfeeding were noted. The patient's HBV DNA level and HBV serological markers were measured with the Roche COBAS TaqMan platform (with the lower limit of detection of $20 \mathrm{IU} / \mathrm{mL}$ ) and Roche Elecsys (Basel, Switzerland). Maternal HBV DNA levels at gestational weeks 24-28 and infant HBV serological markers at 7-12 months were measured at the central laboratory set up by the research group. All other markers were measured at each participating center.

\section{Use of the mobile health application}

A mobile health application called "SHIELD" was developed and used in this study to collect data and provide support for communication between mothers and their doctors. All laboratory test reports, questionnaires and other relevant information was uploaded into SHIELD and made available to mothers and doctors. Mothers could consult with their doctors via SHIELD during the follow-up (Fig. 1).

\section{Outcomes}

The primary outcome was the rate of MTCT following the administration of immunoprophylaxis and antiviral therapy. The secondary outcome was any potential adverse effect noted among infants following antiviral exposure, including birth defect and infant growth. The rate of MTCT was defined as the proportion of infants who were positive for HBsAg, while they had a detectable HBV DNA level when PVST was performed.

\section{Statistical analysis}

Continuous variables are presented as the means \pm standard deviation, and the means are compared by Student's $t$-test or the nonparametric Mann-Whitney $U$ test. Categorical variables are presented as proportions and Pearson's chi-square test or Fisher's exact test were used to compare groups. The association between covariates and the rate of MTCT were assessed with univariate analysis. All statistical tests were two-sided. A $p$-value $<0.05$ was considered statistically significant. All data were analyzed by SPSS 22.0 (IBM Corp., Armonk, NY, USA). 
Yin X. et al: A real-world study of MTCT of HBV
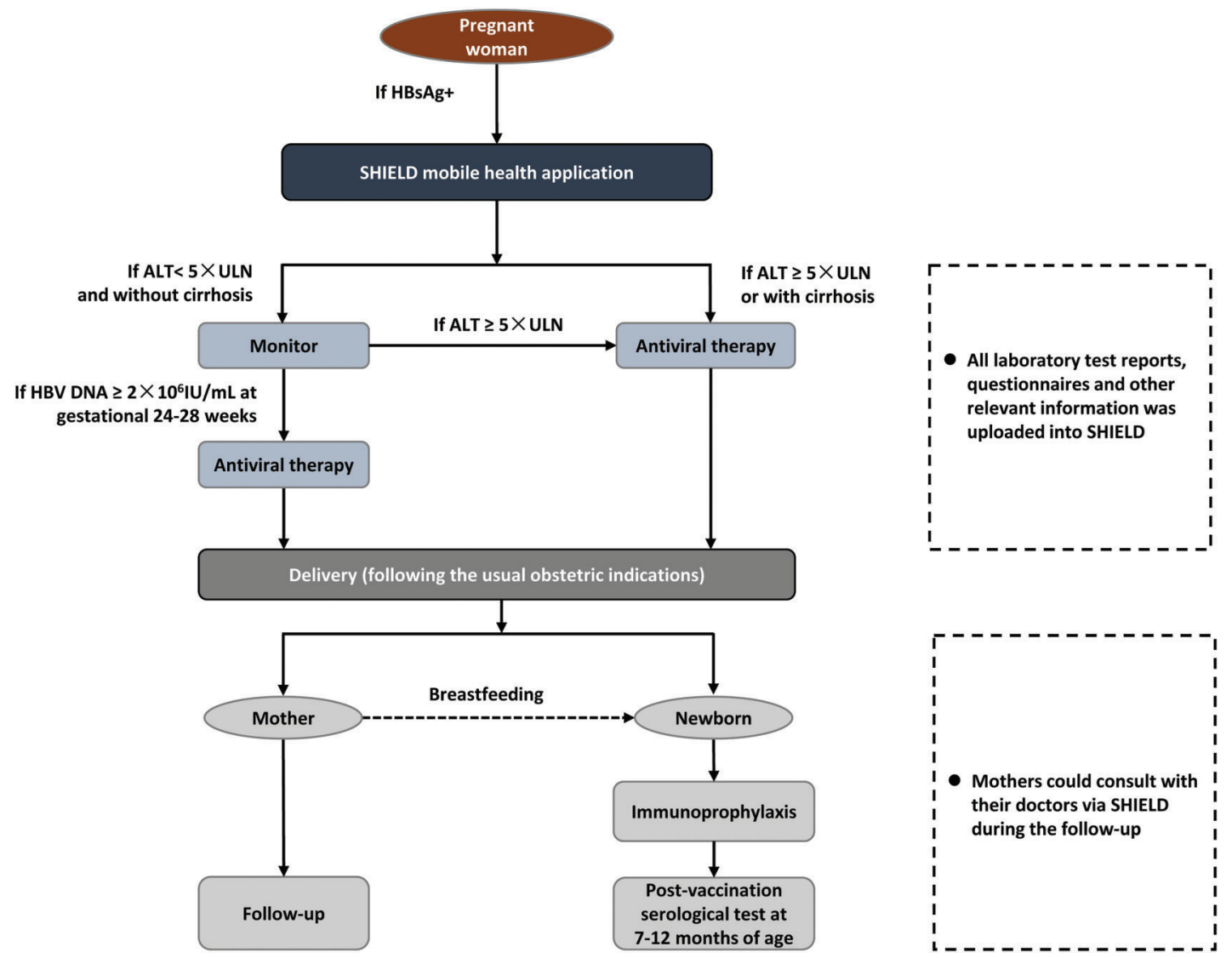

Fig. 1. Use of the mobile health application SHIELD to manage this study.

\section{Ethics approval}

The study was conducted in accordance with Good Clinical Practice, following local regulations and ethical principles described in the Declaration of Helsinki. The study protocol was approved by the ethics committee at each participating center. All authors had access to the study data and reviewed and approved the final manuscript.

\section{Results}

\section{Enrollment and follow-up}

One thousand and twenty HBsAg-positive pregnant women were screened. Among them, 12 pregnant women were excluded due to not meeting the eligibility criteria, 23 pregnant women withdrew informed consent, 11 lost fetuses before delivery, and 69 were lost to follow-up. Therefore, a total of 905 mothers and 924 infants completed followup (Supplementary Fig. 1). The main reasons for loss to follow-up were moving away and parental unwillingness to let their infants undergo blood sampling.

\section{Characteristics of the mothers and infants}

The characteristics of the mothers and infants are listed in Table 1. The distribution of viral load in HBeAg-positive mothers and HBeAg-negative mothers is depicted in Fig. 2.
Up to $73.4 \%$ of HBeAg-positive mothers had an HBV DNA level of more than $6 \log _{10} \mathrm{IU} / \mathrm{mL}$, compared to $6.5 \%$ of HBeAg-negative mothers $(p<0.001)$.

\section{Maternal antiviral therapy}

There were $49.3 \%$ of the mothers enrolled in the study receiving antiviral therapy during pregnancy with either telbivudine (LdT), tenofovir disoproxil fumarate (TDF), or lamivudine (LAM). Among the three nucleot(s)ide analogues, LdT $(76.8 \%)$ was the most frequently used, followed by TDF $(19.9 \%)$ and LAM (3.3\%). Nearly half $(46.4 \%)$ of the 442 mothers received nucleot(s)ide analogues before gestational week 28 , while $49.5 \%$ received between gestational weeks 28 to 32 , and $4.1 \%$ after gestational week 32 . A higher percentage of $\mathrm{HBeAg}$-positive pregnant women received antiviral therapy compared to $\mathrm{HBeAg}$-negative pregnant women $(71.5 \%$ vs. $13.0 \%)$. Up to $72.3 \%$ of 499 pregnant women with an HBV DNA level of more than 2,000,000 IU/mL received antiviral therapy, compared to $21.0 \%$ of those with an HBV DNA level of less than 2,000,000 IU/mL (Supplementary Fig. 2).

\section{Neonatal immunoprophylaxis}

Data on the immunoprophylaxis was obtained from 918 infants whose records were available and complete. The coverage of HepB birth dose and HBIG was $99.8 \%$ and $99.9 \%$, 
Table 1. Characteristics of the HBV-infected pregnant mothers and their infants enrolled in the Shield study, China, 2015-2018

\begin{tabular}{|c|c|}
\hline Characteristics & Total, $n=905$ \\
\hline \multicolumn{2}{|c|}{ Maternal characteristics at baseline } \\
\hline Age in year, mean $\pm S D$ & $28.2 \pm 4.2$ \\
\hline \multicolumn{2}{|l|}{ Alanine aminotransferase } \\
\hline No. of mothers with data* & 892 \\
\hline Mean $\pm S D, U / L$ & $32.2 \pm 42.5$ \\
\hline \multicolumn{2}{|l|}{ Distribution, $n(\%)$} \\
\hline$<1 \times$ ULN, $40 \mathrm{U} / \mathrm{L}$ & $732(82.1)$ \\
\hline$\geq 1 \times$ ULN to $<2 \times$ ULN & $101(11.3)$ \\
\hline$\geq 2 \times$ ULN to $<5 \times$ ULN & $47(5.3)$ \\
\hline$\geq 5 \times$ ULN & $12(1.4)$ \\
\hline \multicolumn{2}{|l|}{ HBV DNA } \\
\hline No. of mothers with data ${ }^{\dagger}$ & 904 \\
\hline $\log _{10}($ HBV DNA), mean $\pm S D$ & $5.7 \pm 2.6$ \\
\hline \multicolumn{2}{|c|}{ Distribution as $\log _{10}$ HBV DNA, $n(\%)$} \\
\hline Undetectable & $38(4.2)$ \\
\hline Detectable to $<6$ & $345(38.2)$ \\
\hline$\geq 6$ to $<8$ & $311(34.4)$ \\
\hline$\geq 8$ & $210(23.2)$ \\
\hline \multicolumn{2}{|l|}{$\mathrm{HBeAg}$} \\
\hline No. of mothers with data ${ }^{\ddagger}$ & 893 \\
\hline \multicolumn{2}{|l|}{ Distribution, $n(\%)$} \\
\hline Negative & $332(37.2)$ \\
\hline Positive & $561(62.8)$ \\
\hline \multicolumn{2}{|c|}{ Infant characteristics at birth, mean \pm SD } \\
\hline Head circumference in $\mathrm{cm}$ & $33.2 \pm 1.3$ \\
\hline Length in cm & $49.8 \pm 1.8$ \\
\hline Birth weight in $\mathrm{kg}$ & $3.2 \pm 0.5$ \\
\hline Apgar score & $9.7 \pm 0.6$ \\
\hline
\end{tabular}

* This category excludes 13 mothers without ALT records.

'This category excludes one mother without HBV DNA data.

${ }^{\ddagger}$ This category excludes 12 mothers without HBeAg records.

Abbreviations: $\mathrm{HBeAg}$, hepatitis B e antigen; HBV, hepatitis B virus; SD, standard deviation; ULN, upper limit of normal.

respectively. The coverage of timely HepB birth dose within 24 $\mathrm{h}$ after birth was $99.7 \%$, and $98.1 \%$ within $12 \mathrm{~h}$ after birth. The coverage of timely HBIG within $24 \mathrm{~h}$ was $99.7 \%$, and $99.1 \%$ within $12 \mathrm{~h}$ after birth. The immunization schedule of four out of ten hospitals involved in this study included two doses of HBIG $100 \mathrm{IU}$, administered to infants born to HBsAg mothers at birth and 1 month of age. In this cohort, $46.6 \%$ of 916 infants received two doses of HBIG 100 IU.

\section{Rate of MTCT}

In total, eight infants were found to be positive for $\mathrm{HBsAg}$ and had detectable HBV DNA after PVST. All eight infants were born to $\mathrm{HBeAg}$-positive mothers with a high viral load. All of them received timely HepB birth dose and HBIG, except for one who was a preterm infant. The rates of MTCT were $0.9 \%$ in $905 \mathrm{HBsAg}$-positive mothers, $1.4 \%$ in 561 mothers who were both HBsAg- and HBeAg-positive mothers, and $1.6 \%$ in 499 mothers who had an HBV DNA level of more than $2,000,000 \mathrm{IU} / \mathrm{mL}$, respectively. The characteristics of the eight infected infants and their mothers are listed in Table 2.

In the 499 mothers with an HBV DNA level of more than $2,000,000 \mathrm{IU} / \mathrm{mL}, 72.3 \%$ received antiviral therapy during late pregnancy. Four HBV-infected infants had maternal antiviral exposure and four were without maternal antiviral exposure. Thus, the rates of MTCT were $1.1 \%$ and $2.9 \%$ in 361 mothers with antiviral therapy and 138 without antiviral exposure, respectively $(p=0.15)$.

\section{Risk factors for MTCT}

Based on the data for the entire cohort, we analyzed the risk factors of MTCT. The results of univariate analyses are listed in Table 3. In the univariate analyses, maternal viral load and $\mathrm{HBeAg}$ status were found to be associated with MTCT. We did not find an association between MTCT and mode of delivery, feeding practice, history of threatened abortion and threatened preterm birth, and history of invasive procedures during pregnancy.

\section{Infant safety outcomes}

Among the liveborn infants, there were no significant differences in the rate of birth defect between infants with maternal antiviral exposure during pregnancy and those without maternal antiviral exposure $(0.5 \%$ vs. $0.7 \%, p=1.00)$. The gestational age, mode of delivery, weight, height, head circumference and Apgar score at birth were similar in the two groups (Table 4).

\section{Discussion}

A real world study enrolling $1008 \mathrm{HBsAg}$-positive mothers and their infants was prospectively conducted to investigate the status quo of MTCT in the real world. The results show that the rate of MTCT among HBV-exposed infants was $0.9 \%$, in the context of high coverage of birth dose immunization and maternal antiviral intervention during pregnancy.

For all infants, free three-dose HepB was provided by the Chinese government, guided by the National Immunization Schedule. In this cohort, the timely HepB birth dose and HBIG were $99.7 \%$ and $99.7 \%$, respectively. In addition, antiviral therapy was administered to $49.3 \%$ of mothers in this cohort. In this study, LdT is the most frequently used NA, followed by TDF and LAM. LdT, LAM and TDF were recommended for maternal antiviral therapy in the previous Chinese chronic hepatitis B guideline. ${ }^{1}$ In June 2014, TDF was approved in China. Before that, LdT and LAM were used in preventing MTCT. $8,13,14$ However, TDF and LdT are preferably recommended for preventing MTCT of HBV, guided by the latest Chinese recommendation, ${ }^{20}$ while TDF is preferably recommended in the American Association for the Study of Liver Diseases and European Association for the Study of the Liver guidelines. ${ }^{16,17}$

Eight infants were found to be infected by HBV who were all born to $\mathrm{HBeAg}$-positive mothers with an HBV DNA level of more than $2,000,000 \mathrm{IU} / \mathrm{mL}$. Four infants were born to mothers who received antiviral therapy and HBV DNA level was less than $5 \log _{10} \mathrm{IU} / \mathrm{mL}$ in two of four mothers and was more than $6 \log _{10} \mathrm{IU} / \mathrm{mL}$ in one of four mothers at delivery. This result indicated that in the real world, even if HBeAgpositive mothers with a high viral load received antiviral therapy and their infants received immunoprophylaxis, perinatal infection cannot be completely prevented. One explanation 
Yin X. et al: A real-world study of MTCT of HBV

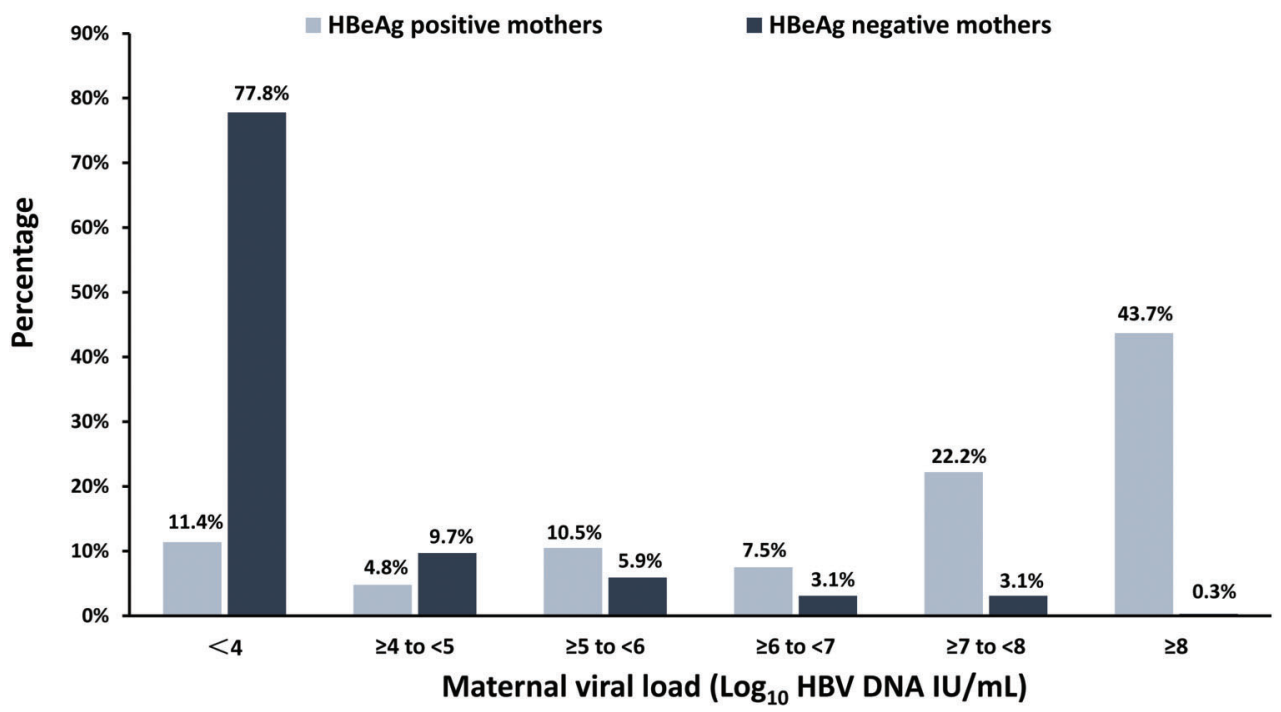

Fig. 2. Viral load distribution in hepatitis $B$ e antigen-positive and hepatitis $B$ e antigen-negative mothers.

for this is that intrauterine infection occurred in the infants. In fact, one of the four infants was found to be positive for HBV DNA at birth, which indicated an intrauterine HBV infection. Another possible explanation is that these infants' infections were caused by vaccine-escape mutants with altered binding capacity for the antibody to $\mathrm{HBsAg} .^{21,22}$ Another four infected infants were born to mothers who had an HBV DNA level of $100,000,000 \mathrm{IU} / \mathrm{mL}$ but without antiviral therapy during pregnancy. One infant did not receive birth dose vaccine due to prematurity. Delayed immunoprophylaxis for premature or low-birthweight $(<2000 \mathrm{~g})$ infants is most likely the cause of these infections. It is recommended that premature infants should also receive timely immunoprophylaxis. ${ }^{23,24}$

In this study, the rates of MTCT were $0.9 \%$ and $1.4 \%$ in $\mathrm{HBsAg}$-positive mothers and in HBsAg- and HBeAg-positive mothers, respectively, which was much lower than the rates reported in previous studies. ${ }^{10,25}$ Besides that, the MTCT rates were $1.1 \%$ and $2.9 \%$ in $\mathrm{HBsAg}$-positive mothers with high viral load with antiviral exposure and those without antiviral exposure, respectively. Although an apparent reduction in the rate of MTCT was found, there was no significant difference in the rates of MTCT between the two groups. The reason may be the low rate of MTCT and that the sample size is not large enough in this study. Similar to our study, a recent study in Thailand demonstrated that the immunoprophylaxis failure rate was $2.0 \%$ in the group without maternal antiviral therapy. ${ }^{7}$

The threshold HBV DNA level for maternal antiviral therapy varies among hepatitis B guidelines. An HBV DNA level of more than $2,000,000 \mathrm{IU} / \mathrm{mL}$ is recommended in the China hepatitis $B$ guidelines as the threshold for antiviral therapy to prevent MTCT, ${ }^{1}$ whereas HBV DNA level of more than 200,000 $\mathrm{IU} / \mathrm{mL}$ has been recommended in the American Association for the Study of Liver Diseases and European Association for the Study of the Liver guidelines. ${ }^{16,17}$ In addition, in Australia and New Zealand, the threshold is set at $10,000,000 \mathrm{IU} / \mathrm{mL} .^{26}$ In this real-world study, all eight HBV-infected infants were born to mothers with HBV DNA level of more than $100,000,000 \mathrm{IU} / \mathrm{mL}$. This result implied that the threshold for antiviral therapy may be higher than the current level in the context of high coverage of timely HepB birth dose and HBIG. On the contrary, in the area where coverage of timely
HepB birth dose and HBIG is low, antiviral therapy during pregnancy might be a compensatory approach for preventing MTCT. Currently, it is important to objectively evaluate the significance of maternal antiviral therapy in preventing MTCT of HBV. Based on immunoprophylaxis, maternal antiviral therapy could be used to complement current prophylaxis in mothers with high viral load to prevent MTCT. On the one hand, we should avoid overuse of maternal antiviral therapy in those who are unlikely to transmit to their infant perinatally. On the other hand, we should do our best to prevent MTCT and its consequent outcomes in HBsAg-positive mothers with a high viral load and in their infants.

Univariate analysis showed that HBeAg positivity and high viral load were strongly associated with MTCT. To identify mothers who have a high risk of MTCT, HBeAg and HBV DNA are the most important factors. Ideally, all HBsAg-positive mothers should have a measurement of HBV DNA levels so that antiviral prophylaxis can be administered to those with a high viral load. However, HBV DNA quantification is expensive and may not be available in remote or under-developed areas due to its cost and technical requirements. In our study, $73.4 \%$ of HBeAg-positive mothers had an HBV DNA level of more than $2,000,000 \mathrm{IU} / \mathrm{mL}$, compared to $6.5 \%$ of $\mathrm{HBeAg}$-negative mothers. Thus, if $\mathrm{HBeAg}$ was utilized to screen high-risk mothers for antiviral therapy, $6.5 \%$ of $\mathrm{HBeAg}$-negative mothers with an HBV DNA level of more than 2,000,000 IU/mL will be missed, whereas $26.6 \%$ of $\mathrm{HBeAg}$-positive mothers with an HBV DNA level of less than 2,000,000 IU/mL will be over-treated. Considering that few infants born to HBsAg-positive and HBeAgnegative mothers would be perinatally infected by HBV after timely immunoprophylaxis at birth, ${ }^{11,25} \mathrm{HBeAg}$ screening might be an acceptable alternative to HBV DNA quantification, especially in areas where HBV DNA testing is unavailable.

The SHIELD mobile application was innovatively developed in this study. First, this new tool was used as an electronic data capture system to collect data including laboratory test reports, immunoprophylaxis and antiviral therapy information, etc. In addition, SHIELD broke down barriers among hepatologists, gynecologists and HBV-infected mothers, providing a platform for communicating without restrictions of time and space. 
Yin X. et al: A real-world study of MTCT of HBV

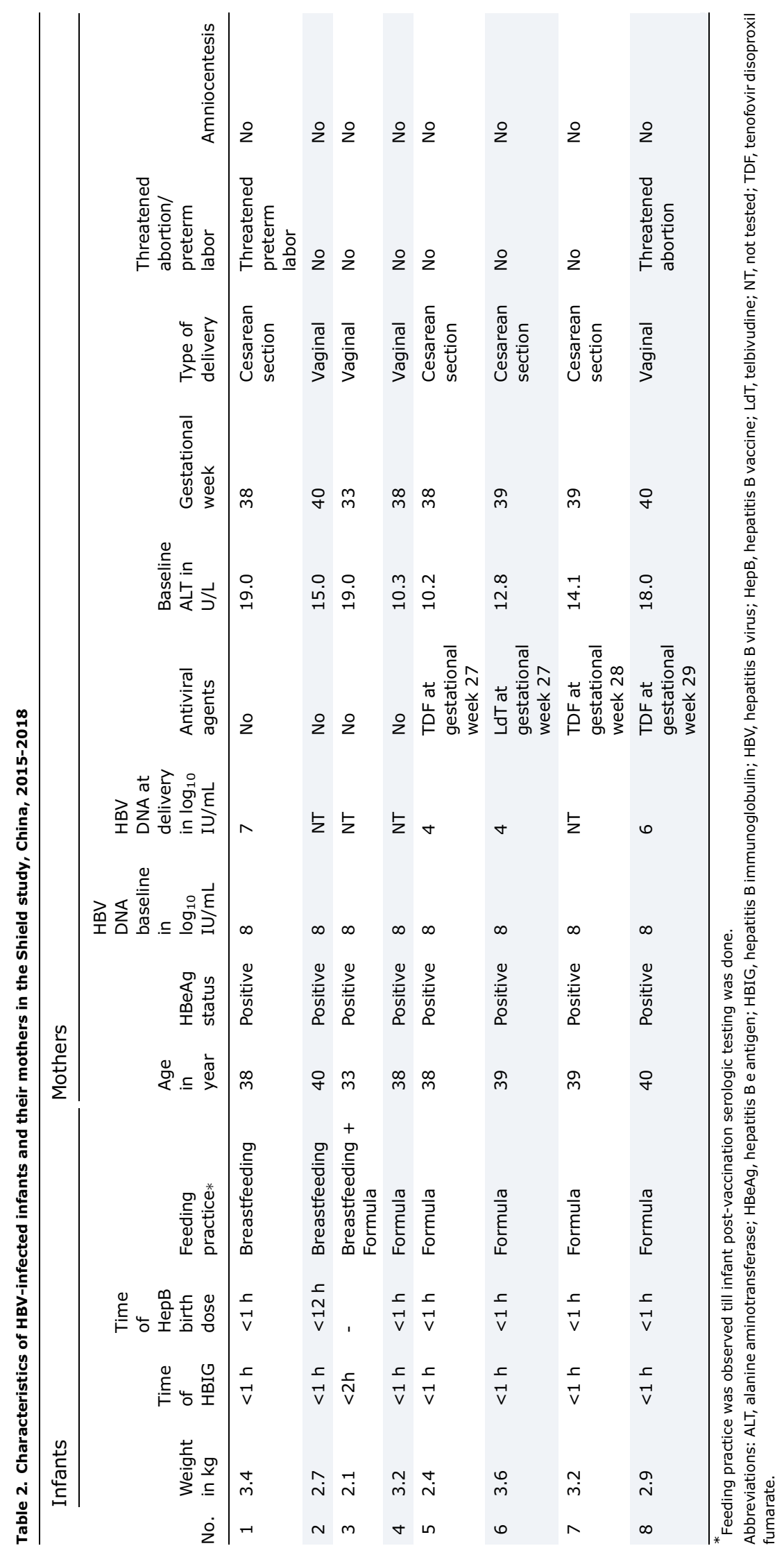


Yin X. et al: A real-world study of MTCT of HBV

Table 3. Univariate analyses on risk factors of mother-to-child transmission

\begin{tabular}{|c|c|c|c|}
\hline \multirow[b]{2}{*}{ Variables } & \multicolumn{2}{|c|}{ Mother-to-child transmission } & \multirow[b]{2}{*}{$p$-value } \\
\hline & Yes & No & \\
\hline Total & $8(0.9 \%)$ & $897(99.1 \%)$ & \\
\hline \multicolumn{3}{|c|}{$\mathrm{HBeAg}$} & 0.03 \\
\hline Positive & $8(1.4 \%)$ & $553(98.6 \%)$ & \\
\hline Negative & $0(0.0 \%)$ & $332(100.0 \%)$ & \\
\hline \multicolumn{3}{|c|}{ HBV DNA level at gestational week 28 in $\log _{10} \mathrm{IU} / \mathrm{mL}$} & 0.002 \\
\hline$<6$ & $1(0.2 \%)$ & $449(99.8 \%)$ & \\
\hline$\geq 6$ to $<8$ & $0(0.0 \%)$ & $183(100.0 \%)$ & \\
\hline$\geq 8$ & $7(2.9 \%)$ & $237(97.1 \%)$ & \\
\hline \multicolumn{3}{|c|}{ Initiation time of antiviral therapy } & 1.00 \\
\hline$<28$ weeks & $2(1.0 \%)$ & $203(99.0 \%)$ & \\
\hline$\geq 28$ weeks to $<32$ weeks & $2(0.9 \%)$ & $215(99.1 \%)$ & \\
\hline$\geq 32$ weeks & $0(0.0 \%)$ & $20(100.0 \%)$ & \\
\hline \multicolumn{3}{|c|}{ Mode of delivery } & 0.54 \\
\hline Vaginal & $4(0.7 \%)$ & $552(99.3 \%)$ & \\
\hline Cesarean section & $4(1.2 \%)$ & $355(98.9 \%)$ & \\
\hline \multicolumn{3}{|c|}{ Feeding practice } & 0.48 \\
\hline Breastfeeding & $3(0.6 \%)$ & $499(99.4 \%)$ & \\
\hline Formula feeding & $5(1.3 \%)$ & $394(98.8 \%)$ & \\
\hline \multicolumn{3}{|c|}{ History of threatened abortion/threatened preterm labor } & 0.28 \\
\hline Yes & $2(1.7 \%)$ & $116(98.3 \%)$ & \\
\hline \multirow[t]{2}{*}{ No } & $6(0.8 \%)$ & $781(99.2 \%)$ & \\
\hline & \multicolumn{2}{|c|}{ History of invasive procedure } & 1.00 \\
\hline Yes & $0(0.0 \%)$ & $7(100.0 \%)$ & \\
\hline No & $8(0.9 \%)$ & $890(99.1 \%)$ & \\
\hline
\end{tabular}

Abbreviations: $\mathrm{HBeAg}$, hepatitis B e antigen; HBV, hepatitis B virus.

SHIELD is exploring a new model of management for preventing MTCT.

The WHO has set the goal to eliminate viral hepatitis as a major public health threat by 2030 , which includes reducing the prevalence of $\mathrm{HBsAg}$ among 5 year-old children to $0.1 \%{ }^{27}$ The WHO Regional Office for the Western Pacific set $2.0 \%$ as the target for eliminating MTCT of HBV. In this study, the rate of MTCT could achieve this target in the real world, in the context of high coverage of birth dose immunization and over $70 \%$ maternal antiviral intervention in mothers with high viral load. The overall coverage of hepatitis $B$ vaccination has increased steadily, having reached $95 \%$ in urban areas in China by $2005 .{ }^{28}$ Besides that, the recommendation for maternal antiviral intervention has been widely accepted. Thanks to the government's policy, the prices of antivirals fell sharply in China, improving the affordability and availability of antivirals. We believe that the 2030 elimination target would be achievable nationwide in the future.

China has achieved major success in the prevention and control of hepatitis $B .^{29}$ To further reduce the rate of MTCT of $\mathrm{HBV}$, actions have already been taken by both government sectors and non-government organizations in China. In December 2017, the National Health Commission (Ministry of Health) officially launched the aptly-named Triple Elimination of MTCT for Hepatitis B, Human Immunodeficiency Virus, and Syphilis Initiative. In July 2015, we launched the Shield Project with the aim of reducing or even eliminating MTCT of HBV. A collaborative network for preventing MTCT has been established

Table 4. Characteristics and birth defects of the infants

\begin{tabular}{llll}
\hline \multirow{2}{*}{ Variable } & \multicolumn{2}{c}{ Maternal antiviral therapy } & \\
\cline { 2 - 3 } & Yes, $n=446$ & No, $n=459$ & p-value \\
\hline $\begin{array}{l}\text { Head circumference } \\
\text { in cm }\end{array}$ & $33.1 \pm 1.3$ & $33.2 \pm 1.3$ & 0.14 \\
$\begin{array}{l}\text { Length in cm } \\
\text { Birth weight in kg }\end{array}$ & $49.9 \pm 1.8$ & $49.8 \pm 1.9$ & 0.05 \\
Apgar score & $9.7 \pm 0.5$ & $3.2 \pm 0.5$ & 0.39 \\
Gestational age in & $39.4 \pm 1.5$ & $9.7 \pm 0.7$ & 0.10 \\
week & $39.1 \pm 1.7$ & 0.12 \\
$\begin{array}{l}\text { No. of cesarean } \\
\text { section }\end{array}$ & 181 & 179 & \\
No. of birth defect & $(40.4 \%)$ & $(38.2 \%)$ & 0.49 \\
& $2(2 / 440$, & $3(3 / 447$, & 1.00 \\
\hline
\end{tabular}


and 29,420 HBsAg-positive mothers and 1630 doctors were enrolled in 124 hospitals nationwide by September 2019. The Shield Project will provide high-level evidence for implementing public health policies to prevent MTCT of HBV and will facilitate the process of eliminating MTCT of HBV in China and globally.

The limitation of this study is that the percentage of HBeAgpositive mothers in this study could not represent the natural distribution in pregnant women with chronic HBV infection due to more $\mathrm{HBeAg}$-positive mothers being willing to participate in our study compared to HBeAg-negative mothers.

In conclusion, The MTCT rate was lower than the WHO Western Pacific Region elimination MTCT target in this realworld study, indicating that a comprehensive management composed of immunoprophylaxis to infants and antiviral prophylaxis to mothers may be a feasible strategy to achieve the 2030 WHO elimination target.

\section{Acknowledgments}

We thank Dr. Lan Zhang for her professional advice on this study, when she worked in the World Health Organization's China Office. We thank all the participants who contributed to the study, with the commitment to elimination of HBV mother-to-child transmission.

\section{Funding}

This work was funded by the China Foundation for Hepatitis Prevention and Control (CFHPC) and National Natural Science Foundation of China (Grant No. 81673243) and the Chinese National Research Grant of the Thirteenth Five-Year Plan for the Key Projects in Infectious Diseases (Grant No. 2017ZX10201201).

\section{Conflict of interest}

JLH has received consulting fees from AbbVie, Arbutus, Bristol Myers Squibb, Gilead Sciences, Johnson \& Johnson, and Roche and has received grants from Bristol Myers Squibb and Johnson \& Johnson. The other authors have no conflict of interests related to this publication.

\section{Author contributions}

Study concept and design (JH and Zhihua Liu), acquisition of data (Zhihua Liu, Zhihong Liu, XY, JH, GH, HZ, MW, WZ, YG, MZ, $\mathrm{XW}, \mathrm{XZ}, \mathrm{GS}, \mathrm{CY}$, and $\mathrm{HL})$, analysis and interpretation of data $(\mathrm{JH}$, Zhihua Liu, and $X Y$ ), drafting of the manuscript (JH, Zhihua Liu, and $X Y$ ), critical revision of the manuscript for important intellectual content (MJ, MJT, SS), administrative, technical, or material support, study supervision (PC, MB, FC, and $\mathrm{HZ}$ ).

\section{References}

[1] Hou J, Wang G, Wang F, Cheng J, Ren H, Zhuang H, et al. Guideline of prevention and treatment for chronic hepatitis B (2015 Update). J Clin Transl Hepatol 2017;5:297-318. doi: 10.14218/JCTH.2016.00019.

[2] Lamberth JR, Reddy SC, Pan JJ, Dasher KJ. Chronic hepatitis B infection in pregnancy. World J Hepatol 2015;7:1233-1237. doi: 10.4254/wjh.v7.i9.1233.

[3] Lavanchy D. Hepatitis B virus epidemiology, disease burden, treatment, and current and emerging prevention and control measures. J Viral Hepat 2004; 11:97-107. doi: 10.1046/j.1365-2893.2003.00487.x.

[4] Wiesen E, Diorditsa S, Li X. Progress towards hepatitis B prevention through vaccination in the Western Pacific, 1990-2014. Vaccine 2016;34:28552862. doi: $10.1016 /$ j.vaccine.2016.03.060.
[5] Chen DS. Hepatitis B vaccination: The key towards elimination and eradication of hepatitis B. J Hepatol 2009;50:805-816. doi: 10.1016/j.jhep.2009.01.002.

[6] Lee C, Gong Y, Brok J, Boxall EH, Gluud C. Hepatitis B immunisation for newborn infants of hepatitis B surface antigen-positive mothers. Cochrane Database Syst Rev 2006:CD004790. doi: 10.1002/14651858.CD004790.pub2.

[7] Jourdain G, Ngo-Giang-Huong N, Harrison L, Decker L, Khamduang W, Tierney C, et al. Tenofovir versus placebo to prevent perinatal transmission of hepatitis $\mathrm{B} . \mathrm{N}$ Engl J Med 2018;378:911-923. doi: 10.1056/NEJMoa1708131.

[8] Zhang H, Pan CQ, Pang Q, Tian R, Yan M, Liu X. Telbivudine or lamivudine use in late pregnancy safely reduces perinatal transmission of hepatitis $B$ virus in real-life practice. Hepatology 2014;60:468-476. doi: 10.1002/hep.27034.

[9] World Health Organization. Eliminating mother to child transmission of HIV hepatitis and syphilis. Available from: https://www.who.int/westernpacific/activities/eliminating-mother-to-child-transmission-of-hiv-hepatitis-syphilis.

[10] Wang F, Zhang G, Zheng H, Miao N, Shen L, Wang F, et al. Post-vaccination serologic testing of infants born to hepatitis B surface antigen positive mothers in 4 provinces of China. Vaccine 2017;35:4229-4235. doi: 10. 1016/j.vaccine.2017.06.019.

[11] Lu Y, Liang XF, Wang FZ, Yan L, Li RC, Li YP, et al. Hepatitis $B$ vaccine alone may be enough for preventing hepatitis $B$ virus transmission in neonates of HBsAg (+)/HBeAg (-) mothers. Vaccine 2017;35:40-45. doi: 10.1016/j. vaccine.2016.11.061.

[12] Cui F, Woodring J, Chan P, Xu F. Considerations of antiviral treatment to interrupt mother-to-child transmission of hepatitis $B$ virus in China. Int J Epidemiol 2018;47:1529-1537. doi: 10.1093/ije/dyy077.

[13] Pan CQ, Han GR, Jiang HX, Zhao W, Cao MK, Wang CM, et al. Telbivudine prevents vertical transmission from $\mathrm{HBeAg}$-positive women with chronic hepatitis $\mathrm{B}$. Clin Gastroenterol Hepatol 2012;10:520-526. doi: 10.1016/j.cgh.2012.01.019.

[14] Han GR, Cao MK, Zhao W, Jiang HX, Wang CM, Bai SF, et al. A prospective and open-label study for the efficacy and safety of telbivudine in pregnancy for the prevention of perinatal transmission of hepatitis B virus infection. J Hepatol 2011;55:1215-1221. doi: 10.1016/j.jhep.2011.02.032.

[15] Pan CQ, Duan Z, Dai E, Zhang S, Han G, Wang Y, et al. Tenofovir to prevent hepatitis B transmission in mothers with high viral load. N Engl J Med 2016; 374:2324-2334. doi: 10.1056/NEJMoa1508660.

[16] EASL 2017 Clinical Practice Guidelines on the management of hepatitis B virus infection. J Hepatol 2017;67:370-398. doi: 10.1016/j.jhep.2017.03.021.

[17] Terrault NA, Lok ASF, McMahon BJ, Chang KM, Hwang JP, Jonas MM, et al. Update on prevention, diagnosis, and treatment of chronic hepatitis $B$ : AASLD 2018 hepatitis B guidance. Hepatology 2018;67:1560-1599. doi: 10.1002/hep.29800.

[18] Sarin SK, Kumar M, Lau GK, Abbas Z, Chan HL, Chen CJ, et al. Asian-Pacific clinical practice guidelines on the management of hepatitis B: a 2015 update. Hepatol Int 2016;10:1-98. doi: 10.1007/s12072-015-9675-4.

[19] Fan R, Yin X, Liu Z, Liu Z, Lau G, Hou J. A hepatitis B-free generation in China: from dream to reality. Lancet Infect Dis 2016;16:1103-1105. doi: 10 . 1016/S1473-3099(16)30327-9.

[20] Hou J, Cui F, Ding Y, Dou X, Duan Z, Han G, et al. Management algorithm for interrupting mother-to-child transmission of hepatitis B virus. Clin Gastroenterol Hepatol 2019;17:1929-1936.e1. doi: 10.1016/j.cgh.2018.10.007.

[21] Carman WF, Zanetti AR, Karayiannis P, Waters J, Manzillo G, Tanzi E, et al. Vaccine-induced escape mutant of hepatitis B virus. Lancet 1990;336:325329. doi: 10.1016/0140-6736(90)91874-a.

[22] Ma $\mathrm{O}$, Wang $\mathrm{Y}$. Comprehensive analysis of the prevalence of hepatitis $B$ virus escape mutations in the major hydrophilic region of surface antigen. J Med Virol 2012;84:198-206. doi: 10.1002/jmv.23183.

[23] Schillie S, Vellozzi C, Reingold A, Harris A, Haber P, Ward JW, et al. Prevention of hepatitis B virus infection in the United States: Recommendations of the advisory committee on immunization practices. MMWR Recomm Rep 2018; 67:1-31. doi: 10.15585/mmwr.rr6701a1.

[24] World Health Organization. Hepatitis B vaccines: WHO position paper, July 2017- Recommendations. Vaccine 2019;37:223-225. doi: 10.1016/j. vaccine.2017.07.046

[25] Chen HL, Lin LH, Hu FC, Lee JT, Lin WT, Yang YJ, et al. Effects of maternal screening and universal immunization to prevent mother-to-infant transmission of HBV. Gastroenterology 2012;142:773-781.e2. doi: 10.1053/j. gastro.2011.12.035.

[26] Wiseman E, Fraser MA, Holden S, Glass A, Kidson BL, Heron LG, et al. Perinatal transmission of hepatitis B virus: an Australian experience. Med J Aust 2009;190:489-492. doi: 10.5694/j.1326-5377.2009.tb02524.x.

[27] World Health Organization. Draft global health sector strategy on viral hepatitis, 2016-2021 - The first of its kind. Available from: http://apps.who. int/gb/ebwha/pdf files/WHA69/A69 32-en.pdf?ua $=1$.

[28] Lu FM, Zhuang H. Prevention of hepatitis B in China: achievements and challenges. Chin Med J (Engl) 2009;122:2925-2927. doi: 10.3760/cma.j. issn.0366-6999.2009.24.001.

[29] Cui $F$, Luo $H$, Wang $F$, Zheng $H$, Gong $X$, Chen $Y$, et al. Evaluation of policies and practices to prevent mother to child transmission of hepatitis $B$ virus in China: results from China GAVI project final evaluation. Vaccine 2013;31 Suppl 9:J36-J42. doi: 10.1016/j.vaccine.2012.11.061. 